\title{
Investigation of Structural Heterogeneity in Individual Amyloid Fibrils using Polarization-resolved Microscopy
}

\author{
Jaladhar Mahato ${ }^{1, \neq, *}$, Korak Kumar Ray ${ }^{1, \neq,+, *}$, Subhadeep Das ${ }^{2}$, Pradeep Kadu², Samir K. \\ $\mathrm{Maji}^{2}$, and Arindam Chowdhury ${ }^{1, *}$ \\ ${ }^{1}$ Department of Chemistry, Indian Institute of Technology Bombay, Mumbai 400076, India. \\ ${ }^{2}$ Department of Biosciences and Bioengineering, , Indian Institute of Technology Bombay, Mumbai 400076, \\ India \\ ${ }^{\dagger}$ Present address: Department of Chemistry, Columbia University, New York, NY 10027, USA
}

$\$$ J.M. and K.K.R. contributed equally to this work.

Corresponding authors

*Email: jaladhar.iitb@gmail.com

*Email: korak.ray@columbia.edu (K.K.R)

*Email: arindam@chem.iitb.ac.in (A.C) 


\section{Supplementary Text}

\section{Derivation for helical anisotropy:}

The following is a derivation of the mathematical expressions that may be used to infer the local structure of an ordered array of fluorophores from the polarization components of their emissions. The experimental setup for which these expressions will be useful consists of fluorescence microscope equipped with a circularly polarized excitation laser and an emission pathway capable of imaging the same field of view along two orthogonal polarizations (referred to from here onward as the $s$ - and $p$-channels). The specific structures for which the expressions given below have been derived are helical amyloid fibers, where the fluorophore binds preferentially to grooves along the individual filaments, and thus the emission dipoles may be assumed to be directed along the length of the filaments themselves (ignoring, at the outset, deviations between the long axis of the fluorophore and the emission dipole and/or binding modes of the fluorophore that do not exactly align it with the length of the filament). As these filaments form a helical bundle in the mature amyloid fiber, the orientation of the ordered array of the fluorophores deviates from the axis of the fiber itself by an amount dependent on the local packing of the filaments and the helical pitch. Assuming helical symmetry, especially over the range of a diffraction-limited spot, the emission dipole's orientation can be seen to follow a conical distribution. ${ }^{1}$ Characterizing this conical distribution will inform on how the fluorophores are oriented locally in the fiber, and consequently, the underlying structural characteristics of the fiber which gave rise to those orientations. The following derivations are ordered in terms of increasing complexity, with progressive additions of features to the emission models being derived.

\section{Case I: The cone is aligned along the s-axis}

For the simplest case, let us assume that the axis of the cone is aligned with the $s$-axis of the polarizing beam splitter (PBS). Let the half-angle of the cone be $\theta$. Let there also be $n$ dipoles in the cone. We can define the angular density of the dipoles as

$$
\bar{\mu}=\frac{n}{2 \pi} .
$$

\footnotetext{
${ }^{1}$ It should be noted the dipoles themselves are not physically forming a cone; they can be more accurately assumed to be arranged on surface of a cylinder. However, the angular distribution of dipoles measured from the fiber axis is the same as those of dipoles arranged in a cone.
} 
The coordinate system is defined such that the $z$-axis is aligned with the conical axis (and thus the $s$-axis of the PBS), and the $x$-axis is along the plane of the image (and is thus aligned with the $p$-axis of the PBS). We will be using spherical coordinates with the angles defined according to the standard convention. Now, consider an infinitesimal arc $d \phi$ at an angle $\phi$ on the cone. The emission intensities for this arc along the $s$ - and $p$-channels are

$$
\begin{gathered}
I_{S}(\phi)=\left|E_{S}(\phi)\right|^{2}=d \phi \bar{\mu} \kappa E_{0}^{2} \cos ^{2} \theta, \\
I_{p}(\phi)=\left|E_{P}(\phi)\right|^{2}=d \phi \bar{\mu} \kappa E_{0}^{2} \sin ^{2} \theta \cos ^{2} \phi,
\end{gathered}
$$

where $E_{0}$ is the electric field associated with a single dipole, and $\kappa$ is an instrument-dependent proportionality constant. Thus, the emissions along the orthogonal polarisation channels for the entire cone can be written as

$$
\begin{gathered}
I_{S}=\int_{0}^{2 \pi} d \phi I_{S}(\phi)=\int_{0}^{2 \pi} d \phi \bar{\mu} \kappa E_{0}^{2} \cos ^{2} \theta=2 \pi \bar{\mu} \kappa E_{0}^{2} \cos ^{2} \theta=n \kappa E_{0}^{2} \cos ^{2} \theta, \\
I_{p}=\int_{0}^{2 \pi} d \phi I_{p}(\phi)=\int_{0}^{2 \pi} d \phi \bar{\mu} \kappa E_{0}^{2} \sin ^{2} \theta \cos ^{2} \phi=\pi \bar{\mu} \kappa E_{0}^{2} \sin ^{2} \theta=\frac{n \kappa E_{0}^{2}}{2} \sin ^{2} \theta .
\end{gathered}
$$

Now, we see that we are left with two equations for $I_{s}$ and $I_{p}$, and two unknowns, $\theta$ and the constant factor $n \kappa E_{0}^{2}$. Measuring $I_{s}$ and $I_{p}$ separately and fitting them to the above equations will yield estimates of both $n \kappa E_{0}^{2}$ and $\theta$. However, in most cases, we only require an estimate of $\theta$ alone. It is obvious, that any ratiometric transformation of $I_{s}$ and $I_{p}$ will remove this dependence of $n \kappa E_{0}^{2}$ and yield a parameter that depends on only $\theta$. Of the many possible options, we have chosen

$$
r_{h}=\frac{I_{s}-I_{p}}{I_{s}+2 I_{p}}=\cos ^{2} \theta-\frac{1}{2} \sin ^{2} \theta=\frac{3 \cos ^{2} \theta-1}{2} .
$$

We note that the definition for the parameter is analogous to the one for fluorescence anisotropy. However, it must be emphasized that this analogy is only mathematical and not conceptual. Nonetheless, we make use of this coincidence in naming our $r_{h}$ parameter helical anisotropy. We see when the conical axis is aligned with the $s$-axis of the PBS, the helical anisotropy $r_{h}$ is sufficient to yield information on the local structure of the underlying fiber. 


\section{Case II: The cone is not aligned with either axis}

While the previous case reduces to a very simple expression for $r_{h}$, it is obvious that, in reality, the situation where the conical axis aligns exactly with the $s$-axis (or, indeed, the $p$-axis) is very unlikely. A more probable scenario is that each individual fiber is arranged at an arbitrary orientation with respect to the $s$-axis. However, since the fibers themselves are long, they can be thought to lie flat on the image plane, which is parallel to the plane defined by the $s$ - and $p$ axes of the PBS. In this case, the deviation between the $s$-axis and the conical axis can be modeled using an in-plane rotation on the image plane. Let $\alpha$ be this angle between the $s$-axis and the conical axis, defined in a clockwise manner. In the reference frame of the cone, defined here by the $s^{\prime}$ - and $p^{\prime}$ - axes, the orthogonal components of the electric field associated with a single dipole oriented at $\theta$ and $\phi$ are identical to the ones in the previous case,

$$
\begin{gathered}
E_{s^{\prime}}(\phi)=E_{0} \cos \theta, \\
E_{p^{\prime}}(\phi)=E_{0} \sin \theta \cos \phi .
\end{gathered}
$$

Thus, in the experimental frame of reference, the components along the $s$ - and $p$-axes (which can be generated by rotating the $s^{\prime}$ - and $p^{\prime}$ - axes counterclockwise by $\alpha$ ) are

$$
\begin{aligned}
& E_{S}(\phi)=E_{s^{\prime}} \cos \alpha-E_{p^{\prime}} \sin \alpha=E_{0} \cos \theta \cos \alpha-E_{0} \sin \theta \sin \alpha \cos \phi \\
& E_{p}(\phi)=E_{s^{\prime}} \sin \alpha+E_{p^{\prime}} \cos \alpha=E_{0} \cos \theta \sin \alpha+E_{0} \sin \theta \cos \alpha \cos \phi .
\end{aligned}
$$

Thus, the associated intensities along these axes are

$$
\begin{aligned}
I_{S} & =\int_{0}^{2 \pi} d \phi \bar{\mu} \kappa\left|E_{S}(\phi)\right|^{2} \\
& =\int_{0}^{2 \pi} d \phi \bar{\mu} \kappa E_{0}^{2}\left(\cos ^{2} \theta \cos ^{2} \alpha+\sin ^{2} \theta \sin ^{2} \alpha \cos ^{2} \phi-2 \cos \theta \cos \alpha \sin \theta \cos \alpha \cos \phi\right) \\
& =2 \pi \bar{\mu} \kappa E_{0}^{2} \cos ^{2} \theta \cos ^{2} \alpha+\pi \bar{\mu} \kappa E_{0}^{2} \sin ^{2} \theta \sin ^{2} \alpha+0 \\
& =n \kappa E_{0}^{2} \cos ^{2} \theta \cos ^{2} \alpha+\frac{n \kappa E_{0}^{2}}{2} \sin ^{2} \theta \sin ^{2} \alpha
\end{aligned}
$$

and 


$$
\begin{aligned}
I_{p} & =\int_{0}^{2 \pi} d \phi \mu\left|E_{p} \overline{(\phi)}\right|^{2} \\
& =\int_{0}^{2 \pi} d \phi \bar{\mu} \kappa E_{0}^{2}\left(\cos ^{2} \theta \sin ^{2} \alpha+\sin ^{2} \theta \cos ^{2} \alpha \cos ^{2} \phi+2 \cos \theta \cos \alpha \sin \theta \cos \alpha \cos \phi\right) \\
& =2 \pi \bar{\mu} \kappa E_{0}^{2} \cos ^{2} \theta \sin ^{2} \alpha+\pi \bar{\mu} \kappa E_{0}^{2} \sin ^{2} \theta \cos ^{2} \alpha+0 \\
& =n \kappa E_{0}^{2} \cos ^{2} \theta \sin ^{2} \alpha+\frac{n \kappa E_{0}^{2}}{2} \sin ^{2} \theta \cos ^{2} \alpha .
\end{aligned}
$$

Our objective is still an expression for the helical anisotropy $r_{h}$, but given the large number of terms involved, it might be simpler to calculate the numerator and denominator separately at first to see if they can be further simplified. Thus,

$$
\begin{aligned}
I_{s}-I_{p} & =n \kappa E_{0}^{2} \cos ^{2} \theta \cos ^{2} \alpha+\frac{n \kappa E_{0}^{2}}{2} \sin ^{2} \theta \sin ^{2} \alpha-n \kappa E_{0}^{2} \cos ^{2} \theta \sin ^{2} \alpha-\frac{n \kappa E_{0}^{2}}{2} \sin ^{2} \theta \cos ^{2} \alpha \\
& =n \kappa E_{0}^{2}\left(\cos ^{2} \theta-\frac{1}{2} \sin ^{2} \theta\right)\left(\cos ^{2} \alpha-\sin ^{2} \alpha\right) \\
& =n \kappa E_{0}^{2} r_{h, i n t} \cos 2 \alpha,
\end{aligned}
$$

and

$$
\begin{aligned}
I_{S}+2 I_{p} & =n \kappa E_{0}^{2} \cos ^{2} \theta \cos ^{2} \alpha+\frac{n \kappa E_{0}^{2}}{2} \sin ^{2} \theta \sin ^{2} \alpha+2 n \kappa E_{0}^{2} \cos ^{2} \theta \sin ^{2} \alpha+n \kappa E_{0}^{2} \sin ^{2} \theta \cos ^{2} \alpha \\
& =n \kappa E_{0}^{2} \cos ^{2} \alpha+n \kappa E_{0}^{2} \sin ^{2} \alpha\left(2 \cos ^{2} \theta+\frac{1}{2} \sin ^{2} \theta\right) \\
& =n \kappa E_{0}^{2} \cos ^{2} \alpha+n \kappa E_{0}^{2} \sin ^{2} \alpha\left(1+\cos ^{2} \theta-\frac{1}{2} \sin ^{2} \theta\right) \\
& =n \kappa E_{0}^{2}\left(1+r_{h, i n t} \sin ^{2} \alpha\right),
\end{aligned}
$$

where $r_{h, \text { int }}=\cos ^{2} \theta-\frac{1}{2} \sin ^{2} \theta$ is the helical anisotropy at $\alpha=0$ that we derived in the previous case. Thus, we see

$$
r_{h, a p p}=\frac{I_{s}-I_{p}}{I_{s}+2 I_{p}}=\frac{r_{h, i n t} \cos 2 \alpha}{1+r_{h, i n t} \sin ^{2} \alpha}
$$


The expression for $r_{h, a p p}$ reveals that it is a function of only $\alpha$ and $\theta$ (in the form of $r_{h, \text { int }}$ ). Thus we see that we do not actually need to measure $I_{s}$ and $I_{p}$ at the exact angle where the $s$ axis is aligned with the fiber (or rather with the conical axis) as in case I. In fact, measuring $I_{S}$ and $I_{p}$ at multiple angles, i.e., at multiple values of $\alpha$, and fitting the resultant values of $r_{h, a p p}$ to the above equation can lead to a more accurate estimation of $r_{h, i n t}$, since it removes the experimental need to exactly measure $r_{h, i n t}$ at a very specific value of $\alpha$.

\section{Properties of $\boldsymbol{r}_{h, a p p}$ as a function of $\alpha$ and $\boldsymbol{r}_{h, i n t}$}

1. Based on the above expression, we can see some symmetries of $r_{h, a p p}$ as a function of $\alpha$. The most trivial of these is

$$
r_{h, a p p}(\alpha)=r_{h, a p p}\left(180^{\circ}+\alpha\right)
$$

This can be interpreted as our measurements being completely insensitive to a $180^{\circ}$ rotation of the PBS. This is because, while we can model the orientation of the ordered array of fluorophores as a cone more or less aligned to the fiber axis, it can be seen that there are in fact two cones (which differ only by a rotation of the conical axis by $180^{\circ}$ ) which can be equivalently used for this purpose. Since $r_{h, a p p}$ has a period of $180^{\circ}$, one can use data from only one $180^{\circ}$ rotation of the PBS to estimate $r_{h, \text { int }}$.

2. A slightly less trivial symmetry is

$$
r_{h, a p p}(\alpha)=r_{h, a p p}\left(180^{\circ}-\alpha\right)
$$

This can be interpreted our measurement not being able to distinguish between two cones oriented at $\alpha$ and $180^{\circ}-\alpha$ (or alternatively, as follows from 1., $\alpha$ and $-\alpha$ ). This arises from the fact that although in these cases, one of the resultant electric fields for one cone along either the $s$ - or $p$-axis is the negative of the other, our measurements are of the resultant intensities, which are only dependent on the magnitudes of the fields and are insensitive to the sign of the fields. Of course, this scenario is only of concern if one only uses data from a single orientation of the PBS (one where the two fibers are reflections of each other along any of the axes). This highlights the need to calculate $r_{h, a p p}$ at different PBS orientations, which completely avoids this scenario. 
3. The behavior of $r_{h, a p p}$ as a function of $\alpha$ changes drastically depending on whether $r_{h, i n t}$ is positive or negative. We will only be considering measurements for the interval $\alpha \in\left[0^{\circ}, 180^{\circ}\right)$ for the following discussion, thereby avoiding issues with periodicity. For $r_{h, i n t}>0$ (i.e., $\theta$ less than approximately $54.7^{\circ}$ ): the maximum value of $r_{h, a p p}$ occurs at $\alpha=0^{\circ}$, and the minimum value of $r_{h, \text { app }}$ occurs at $\alpha=90^{\circ}$.

$$
\begin{gathered}
r_{h, a p p}^{\max }=r_{h, a p p}\left(\alpha=0^{\circ}\right)=r_{h, i n t} \\
r_{h, a p p}^{\min }=r_{h, a p p}\left(\alpha=90^{\circ}\right)=-\frac{r_{h, i n t}}{2}
\end{gathered}
$$

For $r_{h, i n t}<0$ (i.e., $\theta$ greater than approximately $54.7^{\circ}$ ): The maximum value of $r_{h, a p p}$ occurs at $\alpha=90^{\circ}$, and the minimum value of $r_{h, a p p}$ occurs at $\alpha=0^{\circ}$.

$$
\begin{gathered}
r_{h, a p p}^{\max }=r_{h, a p p}\left(\alpha=90^{\circ}\right)=-2 r_{h, i n t} \\
r_{h, a p p}^{\min }=r_{h, a p p}\left(\alpha=0^{\circ}\right)=r_{h, i n t}
\end{gathered}
$$

Thus we see that finding the $r_{h, a p p}^{\max }$ will not always lead to an accurate estimate of $r_{h, i n t}$. For helical bundles which are packed such that the individual filaments have a large deviation from the fiber axis, $r_{h, a p p}^{\min }$ is the accurate estimate of $r_{h, i n t}$. This issue can actually be easily addressed in the following manner. After calculating $r_{h, a p p}$ at different orientations of the PBS, we fit them to the above expression with the bounds that $r_{h, i n t}>0$. This will yield our estimate of $r_{h, \text { int }}$ and the PBS orientation, which yields $\alpha=0^{\circ}$, i.e., where the PBS axis (which we know independently) is aligned with the conical axis. While we may expect some degree of deviation between our conical axis and the fiber axis, our model of the fluorophore distribution necessitates that these two are approximately colinear. Based on our fit of $r_{h, a p p}$, we can verify if this is the case, which it will be if $r_{h, i n t}$ is indeed positive. On the other hand, if the true value of $r_{h, \text { int }}$ is negative, our fit will tell us the cone is arranged at a right angle to our fiber, a situation that is not allowed by our model. In this case, we can correct our estimate of $r_{h, i n t}$ to $-r_{h, a p p}^{\max } / 2$. Thus regardless of the actual value, utilizing our knowledge of the PBS orientation, we can get an accurate estimate of $r_{h, i n t}$. 


\section{Error propagation into $\boldsymbol{r}_{h, \text { int }}$ :}

In general, for $z=f(x, y)$, the error in $x$ and $y$ is propagated to $z$ as

$\sigma_{z}^{2}=\left(\frac{\partial z}{\partial x}\right)^{2} \sigma_{x}^{2}+\left(\frac{\partial z}{\partial y}\right)^{2} \sigma_{y}^{2}+2\left(\frac{\partial z}{\partial x}\right)\left(\frac{\partial z}{\partial y}\right) \operatorname{cov}(x, y)$

where $\sigma$ denotes the standard deviation and cov denotes the covariance. In terms of our experimental quantities, this can be rewritten as

$$
\sigma_{r_{h, a p p}}^{2}=\left(\frac{\partial r_{h, a p p}}{\partial I_{S}}\right)^{2} \sigma_{I_{S}}^{2}+\left(\frac{\partial r_{h, a p p}}{\partial I_{p}}\right)^{2} \sigma_{I_{p}}^{2}+2\left(\frac{\partial r_{h, a p p}}{\partial I_{S}}\right)\left(\frac{\partial r_{h, a p p}}{\partial I_{p}}\right) \operatorname{cov}\left(I_{S}, I_{p}\right)
$$

Since each measurement of $I_{S}$ and $I_{p}$ are paired due to simultaneous measurements in both channels, one can use multiple measurements of the same field of view to compute both the standard deviations and covariance terms in the equation above. From the definition of $r_{h, a p p}$, it follows that

$$
\begin{gathered}
\frac{\partial r_{h, a p p}}{\partial I_{s}}=\frac{\left(1-r_{h, a p p}\right)}{\left(I_{s}+2 I_{p}\right)} \\
\frac{\partial r_{h, a p p}}{\partial I_{p}}=-\frac{\left(1+2 r_{h, a p p}\right)}{\left(I_{s}+2 I_{p}\right)}
\end{gathered}
$$

which can be substituted in the above equation. As can be seen, because of the non-linear relation between $r_{h, a p p}$, and $I_{s}$ and $I_{p}$, the errors in the intensity that is propagated depend on the values of intensities themselves, as well as the value of the noise in the intensities.

The above expression allows us to estimate how much our certainty of $r_{h \text {,app }}$ may change as a result of decay in intensities, caused by, for example, photobleaching of the ThT molecules. If we were to measure $r_{h, a p p}$ for the same FOV, where photobleaching had reduced the intensities such that

$$
I_{s}^{\prime}=m I_{s}, \quad I_{p}^{\prime}=m I_{p}
$$

then, it follows from the definition that the value of $r_{h, a p p}$ remains unchanged. If we assume that the majority of noise for intensities in our system comes from Poisson noise, then it follows the $\sigma \propto \sqrt{I}$, Therefore

$$
\sigma_{I^{\prime} s}^{2}=m \sigma_{I_{s}}^{2}, \quad \sigma_{I^{\prime} p}^{2}=m \sigma_{I_{p}}^{2}, \quad \operatorname{cov}\left(I_{s}^{\prime}, I_{p}^{\prime}\right)=m \operatorname{cov}\left(I_{s}, I_{p}\right)
$$


Additionally,

$$
\begin{gathered}
\frac{\partial{r^{\prime}, a p p}^{\prime}}{\partial I_{s}^{\prime}}=\frac{1}{m} \frac{\partial r_{h, a p p}}{\partial I_{s}} \\
\frac{\partial r_{h, a p p}^{\prime}}{\partial I_{p}^{\prime}}=\frac{1}{m} \frac{\partial r_{h, a p p}}{\partial I_{p}} .
\end{gathered}
$$

Thus, we have

$$
\sigma_{r_{h, a p p}}^{2}=\frac{1}{m} \sigma_{r_{h, a p p}}^{2}
$$

This result signifies that the error in our estimate of $r_{h, a p p}$ follows the same familiar behavior as that of our fluorescent intensities. If the fluorescence intensity doubles, the error in $r_{h, a p p}$ (and indeed, in the intensities) decrease by a factor of $1 / \sqrt{2}$, and vice versa.

\section{Fluorescence imaging at linearly polarized excitation:}

The amyloid-dye binding mode of ThT with these fibrils has been thoroughly studied using polarization-resolved epifluorescence microscopy. Figure S2b shows fluorescence images of two perpendicular $A \beta_{40-42}$ fiber labeled with ThT excited by the linearly polarized light of $405 \mathrm{~nm}$ lasers. It is important to note that the fiber aligned along the incident polarization direction (depicted by double-headed arrow) has substantially more fluorescence intensity (i.e., brighter) than the perpendicular fiber. Furthermore, $90^{\circ}$ rotation of the incident polarization turns the dimmer fibril to the brighter one and vice versa (Figure S2c). This indicates that ThT molecules are roughly oriented parallel to the fiber axis. Such results are in agreement with the earlier theoretical and experimental work. ${ }^{1-4}$ However, we also see non-negligible fluorescence from the fiber perpendicular to the incident polarization, indicating some angular distribution of ThT dipole. 


\section{Supplementary References}

(1) Wu, C.; Wang, Z.; Lei, H.; Duan, Y.; Bowers, M. T.; Shea, J.-E. The Binding of Thioflavin T and Its Neutral Analog BTA-1 to Protofibrils of the Alzheimer's Disease A $316-22$ Peptide Probed by Molecular Dynamics Simulations. J. Mol. Biol. 2008, 384 (3), 718-729.

(2) Krebs, M. R. H.; Bromley, E. H. C.; Donald, A. M. The Binding of Thioflavin-T to Amyloid Fibrils: Localisation and Implications. J. Struct. Biol. 2005, 149 (1), 30-37.

(3) Varela, J. A.; Rodrigues, M.; De, S.; Flagmeier, P.; Gandhi, S.; Dobson, C. M.; Klenerman, D.; Lee, S. F. Optical Structural Analysis of Individual $\alpha$-Synuclein Oligomers. Angew. Chemie - Int. Ed. 2018, 57 (18), 4886-4890.

(4) Groenning, M.; Norrman, M.; Flink, J. M.; van de Weert, M.; Bukrinsky, J. T.; Schluckebier, G.; Frokjaer, S. Binding Mode of Thioflavin T in Insulin Amyloid Fibrils. J. Struct. Biol. 2007, 159 (3), 483-497. 


\section{Supplementary data (Figures)}

a
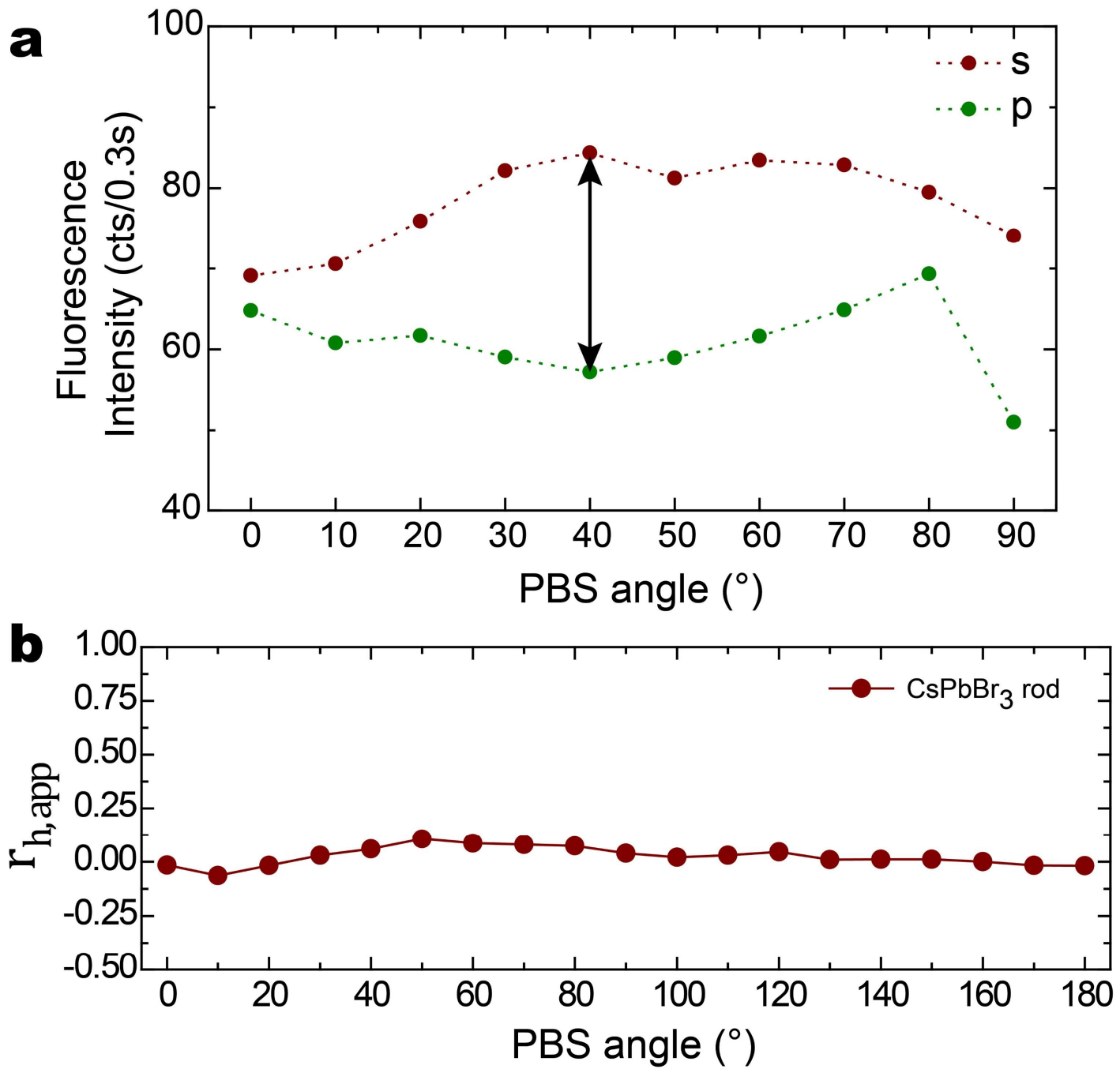

Figure S1. (a) The direction of allowed electric filed in the s-channel calculated from the emission intensity maxima as a function of PBS angle from polystyrene bead using linear polarized excitation. (b) The nominal contribution into the helical anisotropy as estimated from the $r_{h, a p p}$ from sample having isotropic emission (single $\mathrm{CsPBBr}_{3}$ rod) as a function of PBS 


\begin{tabular}{|c|c|c|}
\hline \multirow{3}{*}{ a } & Peptide & Sequence \\
\hline & Fmoc- $A \beta_{40-42}$ & Fmoc-Val-Ile-Ala-OH \\
\hline & Fmoc- $A \beta_{40-41} V$ & Fmoc-Val-Ile-Val-OH \\
\hline
\end{tabular}
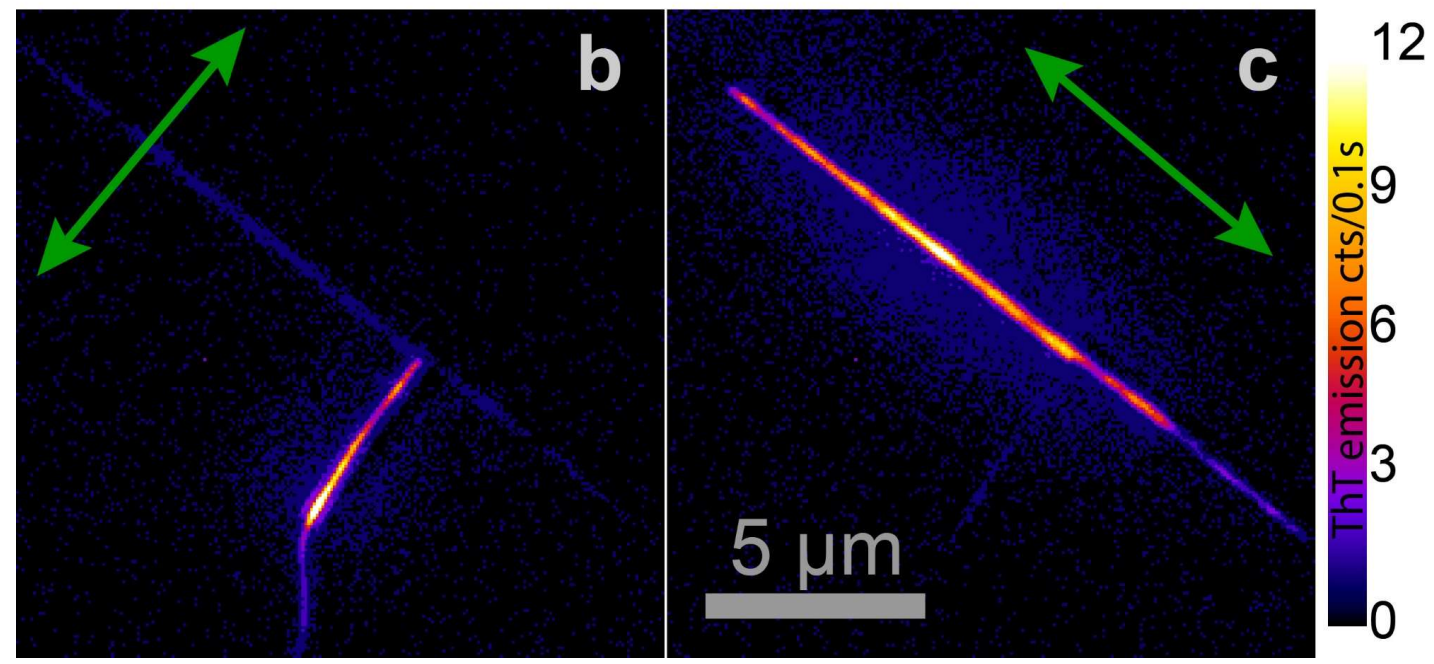

Figure S2. (a) The sequences for the short peptides investigated in the study. $A \beta_{40-4}$ and $\mathrm{A} \beta_{40-41} \mathrm{~V}$ has been designed based on the Alzheimer $\mathrm{A} \beta_{1-42}$ polypeptide. (b, c) Fluorescence images of ThT labeled $A \beta_{40-4}$ fiber when excited by a linearly polarized $405 \mathrm{~nm}$ laser. The direction of the excitation electric field has been shown by the green arrow. 


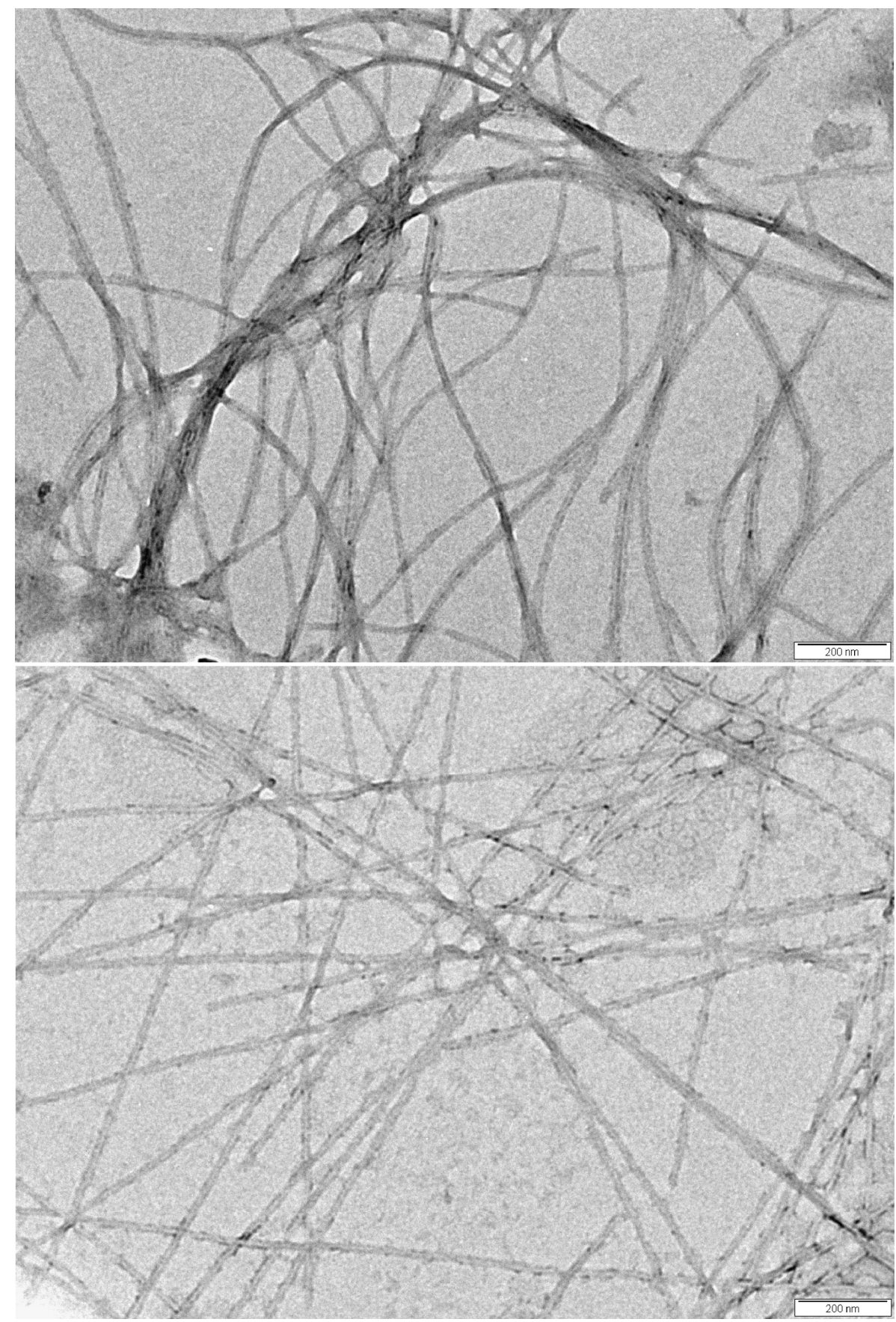

Figure S3. The TEM image of $A \beta_{40-4}$ fibers (top) and $A \beta_{40-41} \mathrm{~V}$ fibers (bottom) depicting the morphological variation. 

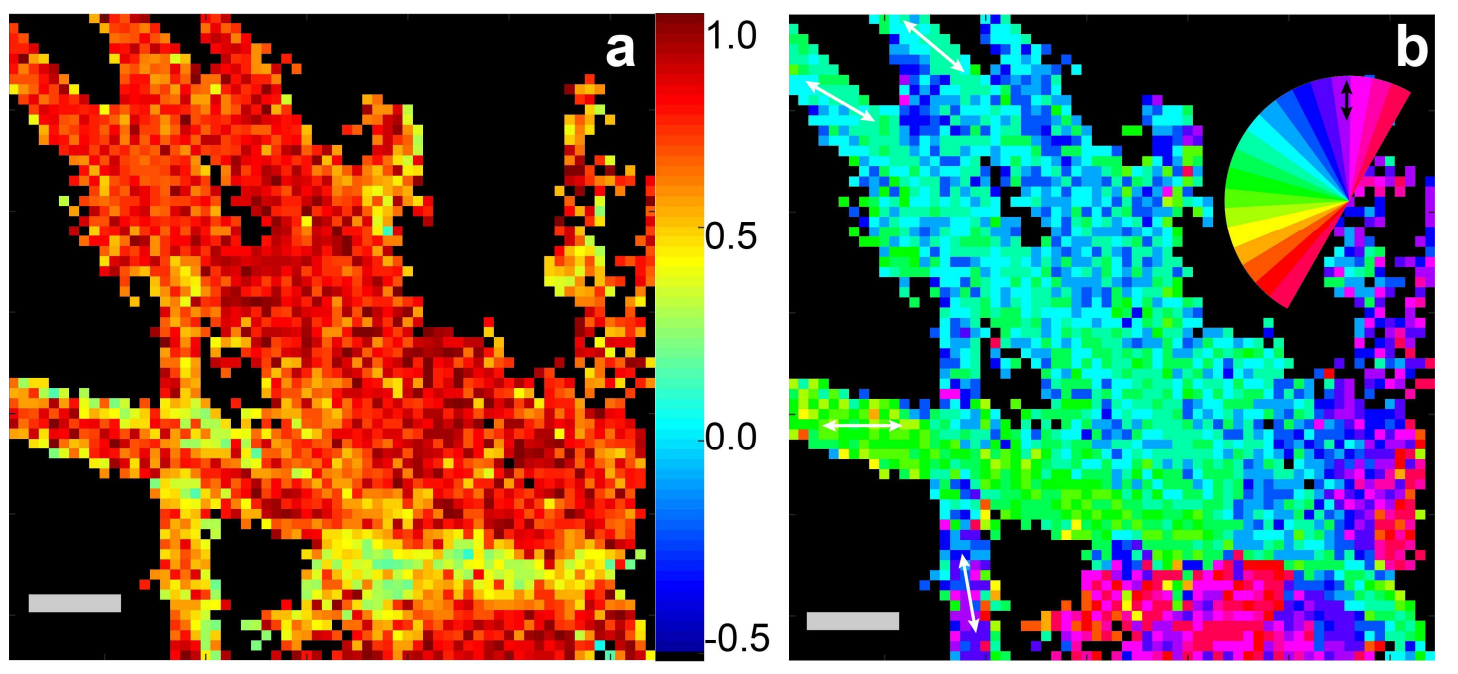

Figure S4. The maximum anisotropy (a) and the cone-axis orientation (b) heatmaps for $\mathrm{A} \beta_{40-4} \mathrm{~V}$. The scale bar in each panel is $500 \mathrm{~nm}$. The cone axis orientation in each pixel is shown by a semicircle color bar similar to Figure 5. 


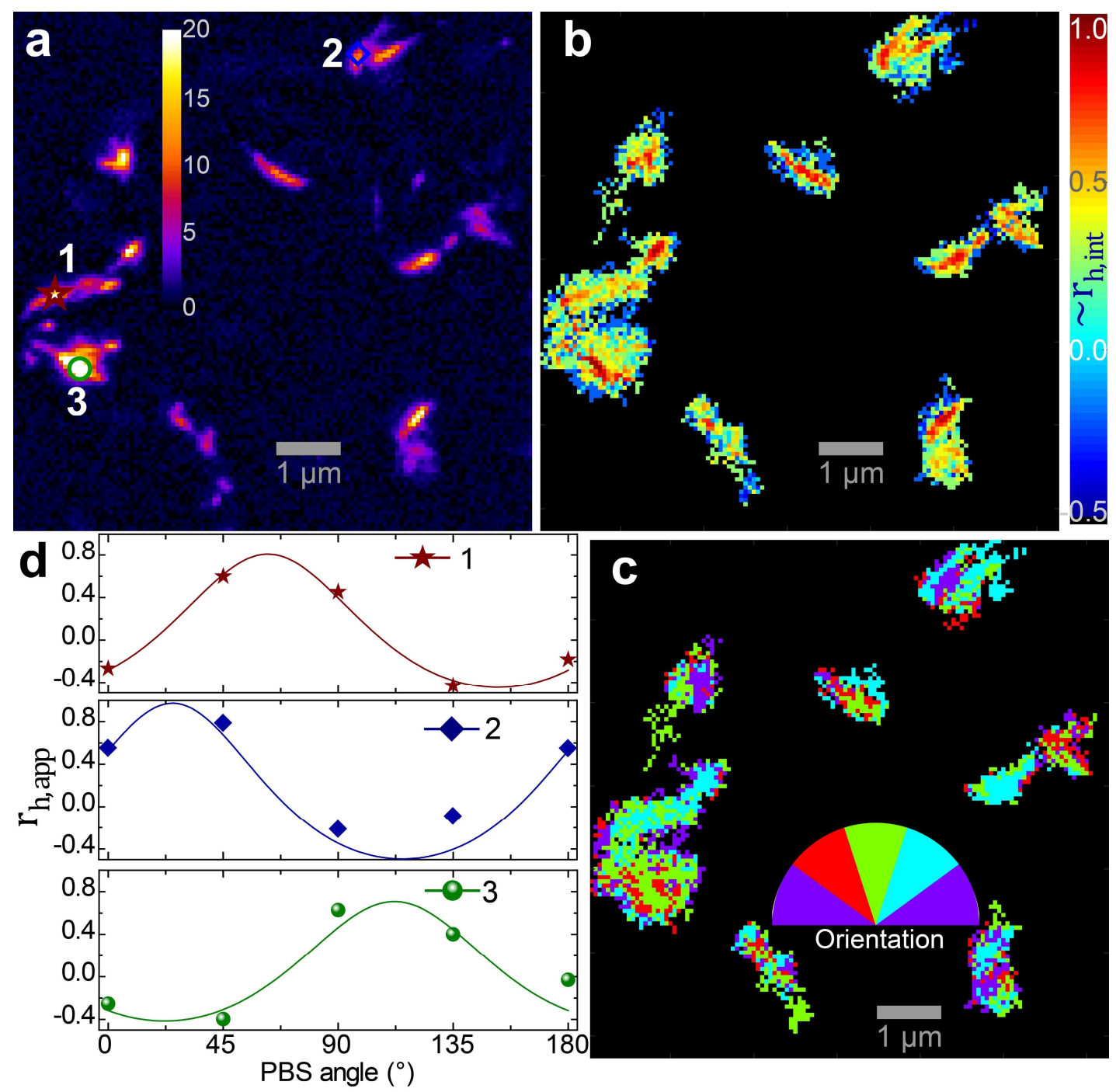

Figure S5. (a) Fluorescence image of $\alpha$-Syn fibril when bound to ThT. (b) The maximum anisotropy heatmap from $s$ - $/ p$ - channel images collected at $45^{\circ}$ rotational increment of the PBS angle. (c) The corresponding cone orientation heatmap, obtained similarly to Figure 5a,b, and Figure S4. (d) The variation of $r_{h, a p p}$ with increase of PBS angle. The data were fitted to the expression for $r_{h, a p p}$. 


\section{Supplementary data (Movie Captions)}

Movie M1: M1, constructed from the images at $s$ channels as a function of $10^{\circ}$ increment of PBS angle.

Movie M2: M2, constructed from the images and $p$ channels as a function of $10^{\circ}$ increment of PBS angle. 